\title{
Discussion on Modern Electronic Information Engineering Technology
}

\author{
Cao Zheng \\ Hubei Three Gorges Polytechnic, Yichang Hubei, 443000, China
}

Keywords: electronic information engineering, modern technology, discussion

\begin{abstract}
With the rapid development of science and technology, we have welcome a fresh era of intelligence, wherein, electronic information engineering technology plays an important role in promoting development of intelligence and technology. In our country, the constant development of electronic information engineering technology determines the development trend of modernization construction. When electronic information engineering technology reaches to a fresh height, it can promote our country to develop towards modernization construction comprehensively to some extent thus to realize great rejuvenation of the Chinese nation. At present, the countries in the world not only compete with each other in military but also in economy and core technology, because all these are important factors to determine the position and speaking right of one country in the world. The development of electronic information engineering technology is still an important reason for various countries to compete with each other, which is also an important basis for the countries to develop towards modernization.
\end{abstract}

Nowadays, electronic information engineering has already penetrated into people's daily life, which always meets with people's increasing demands on spiritual culture and material culture. China is the only country with a large population in the world, and people are using electronic information technology products more and more, which provides a good opportunity for electronic information engineering market, however, there also exists with some problems in the development process of electronic information engineering technology, therefore, it is needed to explore the modern electronic information engineering technology in details to make it conform to the development demand of modern electronic information engineering market in order to promote development of electronic information engineering industry.

\section{Overview of modern electronic information engineering technology}

The computer network technology is adopted to control and manage the electronic information to form a new kind of modern information technology, namely modern electronic information engineering technology. Currently, the modern electronic information engineering technology develops constantly and is widely applied in various levels and fields, which gives full play to its information management function, covering information data supervision, management and storage of electronic information engineering industry. At present, the researches emphases of our country on modern electronic information engineering technology involve the following contents: research on information data supervision, management and storage, research on electronic information transmission and receiving, establishing and perfecting electronic information engineering system. The continuous expansion and innovation of science and technology field promotes the development of electronic information engineering technology in our country and conforms to the development demands of other industries, therefore, it is widely applied in various industries. Besides, the modern electronic information engineering technology can effectively combine with the theoretical knowledge and achievements of other subjects, can realize better innovation thus to further expand its application field. 


\section{Current development situation of electronic information engineering modernization}

\subsection{The electronic information engineering relatively lacks of development space}

The fundamental national situation of our country in current period and imperfect laws and regulations of national government organs have restricted the development of electronic information engineering in our country and caused insufficient development space for it. Though the government and enterprises in our country pay high attention to the development of modern electronic information engineering technology, and they believe the electronic information engineering will have a broad development prospect in the future, there are too many fake commodities in our electronic information engineering market. The electronic information enterprises invest a lot to develop and research the core technology of electronic information engineering products, but the research achievements are randomly stole by other enterprises, which causes large economic loss to the R\&D enterprises. In addition, our country publishes no policies related to introduction of overseas electronic information engineering technology, therefore, the electronic information enterprises need to rely on sufficient funds to develop or introduce related technology by themselves, besides, they face large risks.

\subsection{The electronic information engineering lacks of core technology for development}

Compared with developed countries, our country starts late in developing and researching electronic information engineering technology, besides, our government publishes no policies to protect and support the $R \& D$ achievements of electronic information enterprises, which causes our country to lack of related core technology in developing electronic information engineering technology. The labor cost price in our country is cheap, therefore, most enterprises just rely on cheap labor to help overseas electronic enterprises to produce electronic information products. The electronic enterprises master no core technology, possess no related technology capability and research environment to develop the core technology, therefore, other countries seriously restrict the modernized development of electronic information engineering in our country.

\subsection{The electronic information engineering lacks of innovation}

In recent years, our country has been advocating innovation, it can be seen that our country pays much attention to innovative development of various industries. However, there are few innovations on modern electronic information engineering technology, therefore, the electronic enterprises in our country are appealing the country to publish related policies, but they are restricted by their own fund and enterprise scales, as a result, they are incapable to innovate the modern electronic information engineering technology. What's more, there are so many electronic enterprises in our country, and many industries are still in the initial development stage while our country lacks of funds for modern electronic information engineering innovation, because the electronic information engineering technology needs large number of human powers, materials resources and financial resources.

\section{Specific application of modern electronic information engineering technology}

\subsection{Application of modern electronic information engineering technology in electric appliances}

Nowadays, the modern electronic information engineering technology is developing faster and faster, related technology has been applied in electric appliances. For example, central air-conditioning or refrigerator constant temperature control system an be applied in sewage treatment plant, which is the application presentation combining modern electronic information engineering technology and electric appliances.

\subsection{Application of modern electronic information engineering technology in project cost}

Currently, the database technology in the modern electronic information engineering technology can be applied in project cost effectively. In view of project cost, scientific electronic information 
calculation and processing technology can be effectively applied to analyze data in details, and the obtained conclusion can be taken as the key content for project cost management. Besides, computer network technology can be effectively applied to establish the electronic project cost management platform thus to realize integration of data collection, management, calculation, analysis and application, which can effectively enhance work efficiency and work quality.

\subsection{Application of modern electronic information engineering technology in highway}

For highway industry, the modern electronic information engineering technology is mainly used for enhancing timeliness and correctness of data analysis and data processing, which can establish electronic highway information network platform thus to conduct data analysis and providing information support for managers making correct decisions effectively and enhancing work efficiency.

\section{Modernization development strategy of electronic information engineering}

\subsection{Establish and perfect the laws and systems related to electronic information engineering}

In order to develop the modern electronic information engineering technology, our government should take the lead to formulate and perfect the laws and systems related to electronic information engineering. Wherein, the intellectual property protection should be emphasized, which can not only bring huge economic benefit to electronic enterprises but also is the masterpiece of the R\&D team, effective intellectual property protection is the best confirmation to the electronic enterprises and the $R \& D$ team. Besides, it can effectively stimulate other enterprises' thirst and investment for core technology R\&D. In view of capital support for core technology R\&D, because the core technology $R \& D$ is not featured with certainty and durability, even if most electronic enterprises have already had certain R\&D capability, they dare to research and develop the core technology, therefore, the country should provide capital support, such as implementing more liberal policies on loan situation or interest.

\subsection{Strengthen to cultivate modern electronic information engineering technology talents}

Each industry can not leave from capitals and talents for development. According to related data, the electronic enterprises in China need most modern electronic information engineering technology talents in the world, besides, they have increasing demand on talent quantity, while the electronic enterprises in our country are reducing their satisfaction on talents cultivated from universities, and even face talent shortage problem, which needs our education department to carry out related adjustment scheme on the problem. The universities should strengthen the practical operation capability of the students majoring in electronic information engineering, should add practical operation part into school teaching instead of paying only attention to examination performance. The national government and related education departments should give play to its feature of public trust, publicize practical capability cultivation of universities thus to make students actually focus on their practical ability.

\subsection{Strengthen technology innovation and capital support}

From the perspective of historical development process of various industries, innovation is necessary for better development of modern electronic information engineering technology, wherein, $R \& D$ and innovation of one core technology can drive the modern electronic information engineering technology of whole industry to be a new level. Our government and related departments must encourage various industries to conduct technology innovation, meanwhile, they should publish protection policies on technology innovation and give capital support. For the small-scale electronic enterprises, the national government departments should encourage enterprise cooperation positively, which can enlarge enterprise scale and increase capital for technology innovation thus to stimulate enterprises to conduct technology innovation actively and promote long-term development of modern electronic information engineering technology. 


\subsection{Strengthen effective integration with various industries}

The electronic information engineering industry is not an independent system but must integrate with other industries to create value. Therefore, it is needed to explore the various fields of the electronic information engineering industry to realize its exchange with different departments and fields such as national defense, transportation, highway and even enterprises, and then introduce it into related fields, which can be helpful to serve the society better, effectively research, develop and explore new -type technology according to actual situation thus to effective enhance competitiveness of various industries and actually realize win-win.

\section{Summary}

In summary, electronic information engineering can not only promote further development of science field in our country but also effectively drive the social economy development thus to enhance daily life quality of residents effectively, provide more convenient and faster electronic information products as well as good service for them. Therefore, the electronic information engineering market, residents and the society are all thirsting for the development of electronic information engineering technology. Though there will appear with many problems in the development process of modern electronic information engineering technology, such as poor market environment of electronic information engineering, weak protection ability of laws, lack of support from government and related departments as well as strong market competition, therefore, the country will fully motivate various resources and capabilities, largely support the development of modern electronic information engineering technology thus to drive sustainable development of social economy.

\section{References}

[1] Wan Peng, Cheng Feng. Existing problems in electronic information engineering of our country and improvement measures[J].Electronic Testing, 2017 (6) .

[2] Meng Deqing. Research on modern electronic information engineering technology and its application [J].Electronic world, 2014 (17) .

[3] Mande Riwa. Brief discussions on application measures of computer network technology in electronic information engineering[J].Computer knowledge and technology, 2017 (6) .

[4] Yang Ruoheng. Exploration on modern electronic information engineering technology [J].Diti, 2017 (3) .

[5] Li Chenghao, Yang Guanyu, Tang Shuo. Exploration on modern electronic information engineering technology[J].Communication World, 2016 (5) :13-13,14.

[6] Ye Qing. Research on modern electronic information engineering technology [J]. Information and Computer (theoretical edition), 2016 (10) : 60-61.

[7] Feng Xiaoxi. Exploration on modern electronic information engineering technology [J].Electronic technology and software engineering, 2015 (17) : 263. 